\title{
Atendimento à mulher em situação de violência: construção participativa de um protocolo de trabalho
}

\author{
Assistance to women in situations of violence: participatory \\ construction of a working protocol
}

Ana Pereira dos Santos ${ }^{\mathbf{1}}$ Paula Dias Bevilacqua², Cristiane Magalhães de Melo²

DOI: $10.1590 / 0103-1104202012522$

RESUMO Este relato de experiência narra a construção participativa de um Protocolo de Rede para o enfrentamento das violências contra as mulheres em um município de médio porte no estado de Minas Gerais. Com a ausência de políticas especializadas, trabalhadores/as se organizaram de forma a construir um coletivo dialógico e autônomo que, baseado nas premissas da pesquisa-ação, pudesse propor um instrumento que normatizasse e garantisse atenção competente e intersetorial às mulheres em situação de violência sexual e doméstica. A experiência resultou em um diagnóstico construído de forma coletiva, que apontou os principais desafios institucionais vivenciados pelos serviços. Também, partindo da iniciativa dos profissionais que compuseram o grupo de trabalho, articulada à ampla mobilização da sociedade civil, o Protocolo de Rede foi aprovado como Lei de Iniciativa Popular no município. O trabalho coletivo, solidário e multiprofissional, que deu origem ao documento público, continuou, após aprovação da Lei, por meio de um comitê técnico organizado para acompanhamento do Protocolo de Rede, sustentando um espaço educativo para discussão dos atendimentos, sensibilização e formação permanente dos profissionais.

PALAVRAS-CHAVE Violência doméstica. Educação continuada. Colaboração intersetorial.

1 Instituto René Rachou - Fiocruz Minas - Belo Horizonte (MG), Brasil. anapsant3@gmail.com

2 Universidade Federal de Viçosa (UFV) - Viçosa (MG), Brasil.

\begin{abstract}
This experience reports the participatory construction of a Network Protocol to address violence against women in a medium-sized municipality in Minas Gerais state. Considering a scenario of lack of specialized policies, workers organized themselves in order to build a dialogic and autonomous collective that, based on the premises of action research, could propose an instrument that would regulate and guarantee competent and intersectoral attention to women in situations of sexual and domestic violence. The experience resulted in a diagnosis constructed in a collective way, which pointed out the main institutional challenges experienced by the services. Also, based on the initiative of the professionals who made up the working group, articulated with a broad mobilization of civil society, the Network Protocol was approved as a Popular Initiative Law in the municipality. The collective, solidary and multiprofessional work, which gave rise to the public document, continued, after approval of the Law, through a technical committee organized to follow up the Network Protocol, supporting an educational space for discussion of care, awareness and permanent education of professionals.
\end{abstract}

KEYWORDS Domestic violence. Continuing education. Intersectoral collaboration. 


\section{Introdução}

A partir de 2003, com a criação da Secretaria Especial de Políticas para Mulheres (SPM), firma-se, no Brasil, o compromisso fundamental e inadiável de enfrentamento da violência contra as mulheres. A forma privilegiada para se efetivar os quatro eixos (combate, prevenção, assistência e garantia dos direitos) da Política Nacional de Enfrentamento à Violência contra as Mulheres ${ }^{1}$ tem sido a conformação das redes, que prevê a atuação articulada entre diferentes serviços e equipamentos, públicos ou não, e a comunidade.

No campo da saúde, podem-se enumerar avanços na atenção à mulher em situação de violência, como a elaboração de normas técnicas que tratam do atendimento aos agravos decorrentes da violência sexual ${ }^{2}$, da anticoncepção de emergência ${ }^{3}$, da atenção humanizada às situações da interrupção da gravidez previstas em lei ${ }^{4}$ e sobre o atendimento obrigatório e integral de pessoas em situação de violência sexual em hospitais públicos e conveniados ao Sistema Único de Saúde (SUS) - Lei $n^{0} 12.845^{5}$. No eixo prevenção, cita-se a Política Nacional de Promoção da Saúde, com ações específicas sobre prevenção da violência e estímulo à cultura de paz, e desdobramentos importantes, como a integração da vigilância das violências ao sistema nacional de vigilância em saúde. Especificamente a Portaria $n^{0}$ 104/2011, que, pela primeira vez, definiu a violência doméstica e sexual como agravo de notificação compulsória ${ }^{6}$, trouxe avanços importantes ao dar visibilidade ao fenômeno e por criar mecanismo institucional para dimensionar a magnitude do problema em nível nacional.

No âmbito da assistência social, a Política Nacional de Assistência Social e o Sistema Único de Assistência Social determinam formas de gerir o sistema que demarca o atendimento continuado e familiar a mulheres em situação de violência ${ }^{7}$. No campo jurídico, a
Lei Maria da Penha (Lei ${ }^{\circ}$ 11.340/2006) foi um importante avanço, pois conceitua essa violência como baseada no gênero, além de articular elementos de repressão e prevenção da violência, bem como a responsabilização dos agressores ${ }^{8}$.

Partindo desse cenário, há uma demanda histórica de que esses dispositivos funcionem de forma orgânica, interinstitucional e intersetorial, envolvendo os serviços no reconhecimento da complexidade do evento violência contra a mulher e colocando-os em diálogo. Seja no âmbito da prevenção ou da atenção à mulher em situação de violência, os mecanismos de enfrentamento precisam se afastar cada vez mais da improvisação, avançando no cuidado com a pessoa agredida e, inclusive, evitando formas de violência institucional, como a revitimização da mulher.

Contudo, a lacuna entre as propostas institucionais e a real situação dos serviços de atendimento convoca a pensar em como são acolhidas, nos cotidianos institucionais, as diretrizes e normas elaboradas pelos agentes do Estado e que orientam os processos de trabalho. Se esses dispositivos legais ou normativos não bastam, o que é necessário fazer para que profissionais se sintam engajados a contribuir, em seu fazer profissional, para o fortalecimento de políticas sociais urgentes?

O relato de experiência a seguir teve como objetivo apresentar a trajetória da construção coletiva de um Protocolo de Atenção às Mulheres em Situação de Violência na cidade de Viçosa - MG, um município de médio porte que não conta com serviços especializados de atendimento às mulheres em situação de violência. Trata-se da apresentação do processo de construção participativa de um protocolo de atendimento em rede - Protocolo de Rede (a partir desse ponto, será referido como 'Protocolo'), que envolveu trabalhadores/as dos vários equipamentos públicos que atendem mulheres em situação de violência, descrevendo o processo de organização da iniciativa e seus desdobramentos. 


\section{Material e métodos}

\section{Cenário de estudo}

O município de Viçosa, localizado na região da Zona da Mata do estado de Minas Gerais, possui população de, aproximadamente, 72.220 habitantes, sendo considerado de médio porte, conforme classificação do Instituto Brasileiro de Geografia e Estatística (IBGE) 9 . A história de mobilização em torno do enfrentamento e da proposição de políticas municipais de atenção à mulher em situação de violência se constituiu, principalmente, a partir da reconstituição do Conselho Municipal dos Direitos da Mulher (CMDM), em 2009. No mesmo ano, articularam-se várias ações no município à luz do II Plano Nacional de Políticas para Mulheres ${ }^{10}$ e da Política Nacional de Enfrentamento à Violência Contra as Mulheres $\mathbf{1}$. Entre as ações realizadas, destacam-se um ciclo de debates sobre o tema e um seminário sobre a Lei Maria da Penha, iniciativas pioneiras em uma cidade que timidamente discutia a pauta da violência e que envolveu gestores e trabalhadores das diversas instituições do município.

Considerando as dificuldades institucionais, sociais e políticas do município para o desenvolvimento de ações para o enfrentamento da violência, o CMDM propôs, em 2010, em conjunto com a Câmara Municipal, a criação do Pacto Municipal de Enfrentamento à Violência Contra a Mulher"1, com o objetivo de comprometer os equipamentos municipais e estaduais para ações de enfrentamento à violência. Tal pactuação criou as bases de articulação política para efetivação, também em 2010, do Programa Casa das Mulheres ${ }^{\mathbf{1 2}}$, integrante da Rede de Atendimento de Mulheres em Situação de Violência, em parceria com a Defensoria Pública. O Programa Casa das Mulheres se constituiu como uma atividade de extensão do Departamento de Educação da Universidade Federal de Viçosa (UFV), vinculado ao Núcleo Interdisciplinar de Estudos de Gênero (Nieg), cuja concepção foi idealizada a partir do CMDM. Entre os objetivos do Programa, estão: (i) acolher, informar e orientar mulheres em situação de violência no campo jurídico e psicológico; (ii) articular a rede protetiva às mulheres em situação de violência no município de Viçosa e região, favorecendo o trabalho em rede e mobilizando serviços e equipamentos que contribuam para o enfrentamento à violência contra a mulher; $\mathrm{e}$ (iii) construir um banco de dados sobre a violência contra as mulheres em Viçosa e região.

Considerando o atendimento às mulheres em situação de violência, o município de Viçosa conta com equipamentos básicos de segurança pública, saúde e assistência social. No que se refere à segurança pública, o município possui uma Delegacia de Polícia Militar, uma Delegacia de Polícia Civil e um Posto de Perícia Integrada (PPI). O sistema judiciário é composto pelo Fórum da Comarca de Viçosa, pela Promotoria Pública e pela Defensoria Pública, que atendem Viçosa e cinco outros municípios vizinhos.

No âmbito da saúde, Viçosa é sede da Região de Saúde, que inclui oito municípios. A atenção primária é realizada em Unidades de Saúde da Família (USF) (aproximadamente 62\% de cobertura) e na Policlínica Municipal (atendimento da população não coberta pela atenção primária). O município possui, ainda, um serviço de atenção especializada à saúde da mulher (Centro Estadual de Atenção Especializada Ceae), dois hospitais filantrópicos conveniados ao SUS; além de uma unidade de saúde localizada no Campus da UFV (Divisão de Saúde - DSA/UFV), voltada para o atendimento da comunidade universitária. Adicionalmente, tem-se o Serviço de Vigilância Epidemiológica, vinculado à Secretaria Municipal de Saúde (SVE/SMS), responsável pela gestão da vigilância em saúde do município.

Quanto à Política de Assistência Social, o município conta com o Centro de Referência Especializado em Assistência Social (Creas) e dois Centros de Referência da Assistência Social (Cras), todos localizados na região central. 


\section{Percursos}

Princípios da pesquisa-ação foram utilizados para balizar a construção do Protocolo de Rede de forma participativa e dialógica, valorizando, conforme Minayo ${ }^{\mathbf{1 3}}$, a experiência dos participantes, favorecendo a troca de saberes e a reflexão sobre os temas do cotidiano. A proposta foi operacionalizada com o uso de oficinas em dinâmicas de grupo ${ }^{\mathbf{1 4}}$, estudos de caso, dinâmicas de sensibilização e a técnica Diagrama de Fluxo Causa-Consequência, que, conforme Faria e Ferreira Neto ${ }^{15}$, proporciona que os/as atores sociais analisem sua própria realidade, identificando entraves e propostas para superação de problemas identificados. Adicionalmente, com objetivo de avaliar a implementação do Protocolo, foram realizadas entrevistas coletivas ${ }^{16}$ com integrantes do Comitê Técnico de Acompanhamento do Protocolo de Rede.

Participaram permanentemente do processo quatorze instituições: setor saúde (hospitais, Ceae, SVE/SMS, atenção primária, DSA/UFV), assistência social (Creas, Cras), segurança pública (polícia civil e militar), CMDM, Programa Casa das Mulheres, Câmara dos Vereadores e Defensoria Pública. Aproximadamente 30 pessoas participaram de 10 encontros, sendo que o primeiro consistiu na apresentação dos problemas relacionados ao atendimento às mulheres em situação de violência e pactuação com os/as gestores/as sobre a construção do Protocolo, organizando, em rede, a atenção às mulheres, evitando a revitimização e garantindo atendimento oportuno e mais qualificado.

Os demais encontros foram realizados com trabalhadores/as dos equipamentos, indicados/as pelos/as gestores/as como referência no processo. Tais encontros objetivaram: (i) elaborar diagnóstico participativo da situação dos equipamentos sobre infraestrutura (física, material e humana), capacidade de atendimento às mulheres e superação das dificuldades; (ii) refletir sobre caminhos percorridos pelas mulheres na rede, propondo ações para minimizar a revitimização e oportunizar e qualificar os atendimentos; (iii) propor fluxograma de atendimento em rede; e (iv) propor estratégias para garantir a implementação do Protocolo.

Tais estratégias garantiram um diálogo, não restrito à reflexão racional, mas que envolveu os participantes, considerando os significados afetivos e as vivências relacionadas à violência. Considerou, principalmente, a capacidade de que cada participante se apresentasse como representante de sua instituição ou serviço, podendo discutir as dificuldades ou problemas sem constrangimentos ou julgamentos. O espaço de liberdade, compromisso e protagonismo foi cuidadosamente tecido na elaboração do Protocolo.

Os procedimentos metodológicos foram submetidos e aprovados pelo Comitê de Ética em Pesquisa com Seres Humanos da Universidade Federal de Viçosa, parecer no 277.522.

\section{A construção do Protocolo de Rede}

A construção do Protocolo envolveu duas dimensões principais: técnica e política. A dimensão técnica, primeira etapa do trabalho, consistiu no diagnóstico participativo e na elaboração do Protocolo propriamente dito. A dimensão política envolveu um conjunto de ações e estratégias com vistas a garantir sua implementação.

Na etapa do diagnóstico participativo, foi enfatizada a identificação dos problemas existentes nas instituições e serviços relacionados ao atendimento de mulheres em situação de violência. A elaboração do diagnóstico envolveu, inicialmente, a mobilização da equipe de trabalho utilizando a metodologia de estudo de caso. Elegeu-se para o referido estudo um caso rico em complexidades e problemas, elaborado a partir de um atendimento real realizado por instituições da rede.

$\mathrm{O}$ incentivo à participação que garantisse sinceridade e fidedignidade das informações foi preocupação constante das mediadoras do 
grupo, já que a inibição diante da vigilância do trabalho ou o medo de perseguições ao se falar de entraves políticos definia a postura de muitos integrantes. Tal aspecto se agravava por se tratar de cidade pequena onde as pessoas se relacionavam em outros espaços além do trabalho, assim, conceber o debate como impessoal foi tarefa discutida diversas vezes durante o processo. Por decisão do próprio grupo, não houve rodízio de lideranças, entretanto, a conformação de comissões e subgrupos de trabalho favoreceu a circulação do poder e a diluição do protagonismo entre os participantes.

Entre os problemas levantados, era preocupante e visível a ausência da noção de complementariedade no acompanhamento dos casos. As atribuições dos parceiros da rede de atendimento eram desconhecidas, assim como, na maioria das vezes, o atendimento não era concebido como complexo, multiprofissional e intersetorial. Também foram comuns as queixas relativas à falta de infraestrutura adequada (sala que garantisse sigilo, carro para transporte das mulheres e materiais para uso cotidiano) e de recursos humanos capacitados e comprometidos com o enfrentamento da violência.

No âmbito da saúde, destacaram-se como problemas específicos: (i) ausência de medicamentos (profilaxia das infecções e contracepção de emergência) para os atendimentos de urgência da violência sexual, situação recorrente em todos os equipamentos/serviços de saúde do município; e (ii) falta de sensibilização e/ou capacitação técnica para o atendimento das violências, em especial, a sexual. Especificamente sobre a violência sexual, havia recusa dos profissionais em atenderem e acompanharem os casos, bem como em oferecer laudo circunstanciado, por receio quanto à interface com o judiciário e à possibilidade de serem intimados como testemunhas em futuro processo para apuração do crime. O diagnóstico revelou arranjos grotescos, violência institucional e várias negligências com relação às mulheres, como tempo de espera excessivo, recusa ou precariedade no atendimento e, até mesmo, procedimentos incorretos. A ausência de um protocolo interno, no hospital de referência para atendimento da violência sexual, facilitava a dispersão do caso e o não encaminhamento para a rede de atendimento do município para acompanhamento pós-atendimento de emergência.

Profissionais das USF apresentaram desconhecimento quanto ao acolhimento, atendimento e encaminhamento dos casos de violência, sobretudo a sexual. Entretanto, foi destaque a participação efetiva e engajada de algumas Agentes Comunitárias de Saúde (ACS) na notificação compulsória dos casos de violência doméstica, sexual e outras violências no âmbito da saúde. Tal fato pode estar relacionado a trabalhos anteriores de formação para notificação de casos de violência contra mulheres, coordenados pelo Nieg junto a esse grupo de profissionais.

$\mathrm{Na}$ área da assistência social, não havia a clareza de que os casos precisavam ser referenciados, avaliados e acompanhados de acordo com a necessidade de cada família. A perspectiva do acompanhamento familiar, do diagnóstico de vulnerabilidades e riscos sociais para as crianças e outros membros a partir da violência sofrida pela mãe foi um tema bastante discutido no grupo. Além disso, a falta de equipamentos de apoio, como abrigo que acolhesse provisoriamente a mulher e seus filhos para situações de emergência dificultava sobremaneira o trabalho das equipes. Não havia qualquer canal de comunicação com os serviços de saúde, e a falta de instrumentais para acompanhamento, referência e contrarreferência dos casos isolava ainda mais os serviços da assistência social dos demais equipamentos da rede.

A área da segurança pública, sobretudo a Polícia Civil, apresentou-se como referência principal para a população, mas, na mesma proporção, mostrou-se isolada e resistente ao trabalho coletivo e em rede. Entretanto, apresentou clara disposição em construir, junto com o hospital de referência, uma 
forma de garantir o registro das provas e facilitar o exame de corpo de delito, já que nessa época ainda não havia sido implantado no município o PPI. Ainda no âmbito da segurança pública, a participação da Polícia Militar se mostrou inexpressiva, sendo que seus representantes abandonaram os encontros antes da conclusão do processo.

Sobre o Poder Judiciário, apesar de sua importância na implementação da Lei Maria da Penha, mostrou-se equivocado e negligente no julgamento dos casos pautados na referida lei. Não estabeleceu diálogo com o grupo e tampouco favoreceu qualquer mudança interna que contemplasse as novidades trazidas pelo Protocolo. Ainda hoje, o município sofre com arbitrariedades e equívocos advindos dos processos julgados no Poder Judiciário local.

A Defensoria Pública apresentou competência técnica no acompanhamento dos casos de violência contra a mulher. No diagnóstico, ficou claro seu papel de protagonista no atendimento oportuno e adequado, além da sensibilidade e da militância, em consonância com o movimento feminista e com as leituras que contemplam a categoria gênero na execução do trabalho. Já o Programa Casa das Mulheres, pela interface com a Universidade, bagagem teórica e militância política, acolheu o lugar de referência nas discussões, garantindo a coesão necessária para os primeiros momentos do grupo.

À medida que os problemas eram compartilhados, emergia um sentimento de segurança e compreensão entre os participantes. Enquanto se aproximavam através da partilha dos problemas e dos desafios, eram encorajados a pensar soluções possíveis e que estivessem na governabilidade dos participantes. Assim, evitaram-se os sentimentos de impotência e desencorajamento tão comuns nos fóruns e discussões sobre políticas públicas. As queixas, em sua maioria, apontavam falta de capacitação e investimento no trabalho. Mas, também, acentuada solidão e inibição para se pensar o problema em sua complexidade e abrangência.

A evasão foi um problema enfrentado durante a construção do Protocolo, a qual, em parte, ocorreu devido ao amadorismo em participar e sustentar espaços de discussão que não tivessem viés institucional e não fossem formalizados e enquadrados dentro de decisões políticas advindas do topo da hierarquia. Nesse sentido, balizaram a coordenação do grupo as perspectivas teóricas da análise institucional17, cuja compreensão das instituições passa pela dialética entre as forças instituídas (determinações, normativas) e as forças instituintes (movimentos inventivos e nômades). A partir desse olhar, o grupo foi convidado a permanecer no lugar marginal do não instituído, fora, portanto, da rigidez das instituições às quais os atores pertenciam. Nessa posição, entende-se que a criatividade e a inventividade puderam ser potencializadas, emergindo, inclusive, a coesão necessária para que o grupo continuasse a existir, já que não estava formalmente instituído.

Assim, considera-se que o processo de construção do Protocolo foi uma novidade, e o protagonismo dos atores envolvidos precisava ser, aos poucos, assumido como dimensão de liberdade e responsabilidade pelo trabalho e pelo coletivo. Também, precisava ganhar espaço a possibilidade de pensar que um movimento, a priori, não legitimado institucionalmente, poderia ter força e potência à medida que o grupo fosse sendo fortalecido entre seus participantes. Um movimento político nômade que foi incorporando em sua composição um viés de respeito e ganhando atenção das instituições à medida que qualificava sua discussão e fortalecia seus membros para o enfrentamento da violência contra as mulheres.

Partindo do pressuposto de que refletir não está dissociado do agir ${ }^{\mathbf{1 8}}$, a problematização e a reflexão coletivas sobre fragilidades e potencialidades das instituições e dos equipamentos estabeleceram condições para que os participantes produzissem conhecimento crítico e engajado com as mudanças necessárias para consolidação da rede de atendimento. O diagnóstico participativo, assim como as trocas de experiências advindas do próprio processo, criou condições para uma ampla avaliação 
da rede em Viçosa, bem como dos caminhos percorridos pelas mulheres na busca de atendimento. Esses resultados foram fundamentais para a proposição do Protocolo e significativos quando avaliado o percurso de trabalho.

As dificuldades de comunicação entre as instituições, além da necessidade de garantir agilidade nos atendimentos e continuidade do cuidado, sobretudo nos casos de violência sexual, fizeram emergir a necessidade de desenvolvimento de um dispositivo on-line, denominado 'protocolo de atendimento online', para registro de informações individuais sobre a pessoa atendida, história relatada de violência e procedimentos realizados por cada instituição que atendeu a mulher. Com esse dispositivo, considera-se que a violência institucional poderia ser minimizada, pois não haveria necessidade de a mulher recontar a história de violência sofrida em cada serviço em que fosse atendida. O acesso à ficha é restrito aos serviços, respeitando o sigilo e o princípio ético de privacidade.

O Protocolo foi, assim, organizado de forma a possibilitar não só a compreensão do que é violência, como também as atribuições das diversas instituições no atendimento dos casos. Além disso, contemplou a elaboração e a pactuação do fluxograma de atendimento em rede para encaminhamento das mulheres. Para tanto, foram definidas 'portas de entrada' dos casos, sendo pactuado que o hospital municipal referência em maternidade e ginecologia seria o primeiro encaminhamento nos casos de violência sexual. Para as demais formas de violência, qualquer instituição poderia atuar como porta de entrada, sendo os encaminhamentos realizados de acordo com as demandas das mulheres.

Em sua dimensão política, o processo de construção do Protocolo envolveu a realização de uma Audiência Pública na Câmara dos Vereadores, dando visibilidade tanto aos problemas enfrentados cotidianamente pelas mulheres que procuravam os serviços quanto à precariedade de recursos e orçamentos vivida pelos trabalhadores. A audiência se mostrou valorosa pelo constrangimento que causou às figuras políticas e do poder executivo local diante das gravidades ali relatadas e pelo compromisso firmado entre a Câmara dos Vereadores e o grupo de construção do Protocolo. Após a audiência, houve uma série de reuniões com os serviços, sobretudo aqueles que ainda permaneciam resistentes à proposta, e diversas pactuações foram refeitas ou atualizadas.

Além da Audiência Pública, foram feitas mobilizações junto à população com o intuito de propor uma lei de iniciativa popular que tratasse do atendimento em rede e da implementação, pelo município, do Protocolo. Foram coletadas mais de três mil assinaturas e, em outubro de 2014, foi aprovada a Lei municipal no 2.417, conhecida como 'Lei do Protocolo'19, com o intuito de reforçar as leis e normativas estaduais e nacionais, comprometendo o poder público municipal com a causa das mulheres, mantendo a pauta na agenda pública, sobretudo no que diz respeito à garantia da qualificação do atendimento às situações de violência.

O documento final foi organizado em três partes $^{\mathbf{2 0}}$ : (i) bases teóricas e conceituais para compreensão da violência contra as mulheres, aspectos epidemiológicos e sinais de alerta para situações de risco; (ii) atribuições das diversas instituições que atendem os casos, incluindo especificidades, aspectos éticos e público alvo, contemplando, inclusive, os nove municípios integrantes da Região de Saúde Viçosa; e (iii) proposta de educação permanente para trabalhadores/as e demais agentes de enfrentamento que compõem a rede de atendimento.

\section{Desdobramentos de um trabalho em permanente construção}

Após sancionada a 'Lei do Protocolo', como desdobramentos do trabalho, foi criado: o Comitê Técnico de Acompanhamento do Protocolo, sendo esse um fórum permanente de discussão dos casos atendidos na rede, de avaliação da operacionalização dos fluxogramas de atendimento e de fiscalização do 
cumprimento do Protocolo. O comitê objetivava manter e fortalecer o compromisso das instituições e dos serviços com a continuidade e a qualidade do atendimento às mulheres.

O comitê técnico foi organizado cerca de um ano após o início das atividades e teve como primeira tarefa sistematizar os processos de trabalho internos de atendimento às mulheres em situação de violência, considerando os fluxos interinstitucionais e demais orientações previstas no Protocolo. Vale ressaltar que o trabalho foi desenvolvido com empenho pelas profissionais envolvidas, domínio das atribuições de cada equipamento da rede, cuidados com a não revitimização institucional e disposição para a articulação das ações em rede.

Analisou-se que a causa dessa dinamização do grupo se deu em função de três fatores. O primeiro foi que a equipe do Hospital São Sebastião, responsável pelo primeiro atendimento de casos de violência sexual, estava naquele momento constituída por um grupo sensível à responsabilidade no atendimento e enfrentamento à violência contra as mulheres. Dada a promulgação da Lei $n^{0} 12.845$, que dispõe sobre o atendimento obrigatório e integral de pessoas em situação de violência sexual, como já citado, a equipe havia sido capacitada para o atendimento integral da mulher, incluindo a coleta de material para o trabalho da perícia policial. Diante desse cenário, criou-se um dispositivo político, já que o atendimento emergencial da saúde é um elemento central tanto para que haja acolhimento da pessoa vitimada quanto para desencadear o procedimento judicial referente ao crime de violência.

O segundo fator foi a implantação do Posto de Perícia Integrada em Viçosa (PPI), facilitando o procedimento judicial dos crimes de violência. Essa infraestrutura criou um cenário social de apoio ao trabalho em rede, além de um ambiente subjetivo de reconhecimento e respeito ao empreendimento das profissionais envolvidas. O terceiro e último fator foi a própria transformação do Protocolo em lei municipal, conferindo dimensão política e institucional ao trabalho em rede e contribuindo para a criação de um horizonte de otimismo quanto à efetividade das ações empreendidas.

Em 2015, foi realizado um seminário municipal para socialização, entre os/as trabalhadores/as, dos processos de trabalho internos para atendimento elaborados por cada instituição da rede, além da apresentação do trabalho para o público em geral, publicizando os desdobramentos da lei municipal. Atualmente, o Comitê Técnico de Acompanhamento do Protocolo sustenta, por meio do diálogo intersetorial e permanente, a implementação e continuidade do Protocolo. São realizadas reuniões mensais com objetivo de acompanhar e avaliar os atendimentos na rede, propondo medidas corretivas quando da identificação de problemas. Além disso, o Comitê mantém os/ as profissionais alertas e sensíveis às ações de enfrentamento das violências e a rede articulada para atendimento oportuno e adequado às mulheres.

\section{Considerações finais}

Implementado no município a partir de uma lei municipal de iniciativa popular, a experiência de construção do Protocolo foi exitosa e particular por se valer da capacidade de organização dos próprios trabalhadores diante da ausência de serviços especializados de atendimento às violências contra mulheres e de um trabalho em rede que fosse humano e complementar o suficiente para dar conta, de forma oportuna e qualificada, dos casos ocorridos no município. O movimento de construção do Protocolo, portanto, teve início de forma improvisada, sem organização institucional e baseado, exclusivamente, na vontade e preocupação dos profissionais com as mulheres que atendiam. Por muito tempo, o grupo de trabalho sobreviveu dessa forma. Foi com esse caráter, inclusive, que manteve uma forte coesão. No decorrer do processo, a necessidade de institucionalização das ações, a fim de que pudessem permanecer na agenda de 
enfrentamento da violência contra as mulheres no município, mostrou-se imperativa.

Apropriados da proposta, o processo de investigação junto com os trabalhadores produziu, além do documento do Protocolo, um diagnóstico participativo que deu condições para uma ampla avaliação da rede de proteção às mulheres no município, assim como textos, parcerias e experiências advindos do próprio processo, que são muito significativos quando se avalia o percurso de trabalho.

O processo de construção do Protocolo contribuiu significativamente para a consolidação de uma rede não especializada de enfrentamento da violência contra as mulheres, respondendo às exigências atuais de organização dos atendimentos para esses casos de violência. Além disso, ter como eixo norteador a perspectiva do trabalho solidário, complementar e em rede favoreceu a interlocução entre os parceiros e a construção de estratégias de mobilização que potencializam a atenção integral aos casos.

$O$ viés educativo que pautou a proposta proporcionou um espaço coletivo, democrático, de formação e debate sobre o tema. Como não há garantias de continuidade das políticas de enfrentamento das violências contra as mulheres no Brasil, acredita-se que esse compromisso dependerá, também, da sensibilidade, disposição técnica e do comprometimento político de cada um dos envolvidos com as políticas públicas do município. São os atores institucionais que, mobilizados, colocam os documentos em movimento, criam espaços de debate e confrontam os problemas. A mudança que se espera ver depende, essencialmente, da mobilização dos trabalhadores para com essa violação de direitos e da disposição de se comprometerem, em parceria com a comunidade e com o poder público local, com novas e melhores estratégias de acolhimento, atendimento e acompanhamento das mulheres em situação de violência.

\section{Agradecimentos}

Aos/Às profissionais dos serviços da rede de atenção às mulheres em situação de violência do município de Viçosa (MG). À Fundação de Amparo à Pesquisa do Estado de Minas Gerais (Fapemig) (Processo no CDS - APQ-03625-12) e ao Conselho Nacional de Desenvolvimento Científico e Tecnológico (CNPq) (Processo $\mathrm{n}^{\mathrm{o}} 404877 / 2012-5$ ), pelo apoio financeiro à pesquisa e pela concessão de bolsas de estudos.

\section{Colaboradoras}

Santos AP (0000-0003-2758-845X)* e Melo CM (0000-0002-2817-6759)* contribuíram para a construção e a análise dos dados e a elaboração, revisão crítica e final do conteúdo do manuscrito. Bevilacqua PD (0000-00030015-2154)* contribuiu para a análise dos dados e a elaboração, revisão crítica e final do conteúdo do manuscrito. 


\section{Referências}

1. Brasil. Presidência da República, Secretaria Nacional de Enfrentamento à Violência contra as Mulheres; Secretaria de Políticas para as Mulheres. Política Nacional de enfrentamento da violência contra as mulheres. Brasília, DF: Secretaria Nacional de Enfrentamento à Violência contra as Mulheres; Secretaria de Políticas para Mulheres; 2011.

2. Brasil. Ministério da Saúde, Secretaria de Atenção à Saúde, Departamento de Ações Programáticas Estratégicas, Área Técnica de Saúde da Mulher. Prevenção e tratamento dos agravos resultantes da violência sexual contra mulheres e adolescentes: norma técnica. Brasília, DF: Ministério da Saúde; 2005.

3. Brasil. Ministério da Saúde, Secretaria de Atenção à Saúde, Departamento de Ações Programáticas Estratégicas, Área Técnica de Saúde da Mulher. Anticoncepção de Emergência: perguntas e respostas para profissionais de saúde. Brasília, DF: Ministério da Saúde; 2005

4. Brasil. Ministério da Saúde. Secretaria de Atenção à Saúde, Departamento de Ações Programáticas Estratégicas, Área Técnica de Saúde da Mulher. Atenção Humanizada ao Abortamento: norma técnica. Brasília, DF: Ministério da Saúde; 2005.

5. Brasil. Lei $\mathrm{n}^{\circ} 12.845$, de $1^{\circ}$ de agosto de 2013. Dispõe sobre o atendimento obrigatório e integral de pessoas em situação de violência sexual. [acesso em 2019 ago 14]. Disponível em: https://www2.camara.leg.br/ legin/fed/lei/2013/lei-12845-1-agosto-2013-776663 publicacaooriginal-140646-pl.html.

6. Brasil. Ministério da Saúde. Portaria ${ }^{\circ}$ 104, de 25 de janeiro de 2011. Define as terminologias adotadas em legislação nacional, conforme o disposto no Regulamento Sanitário Internacional 2005 (RSI 2005), a relação de doenças, agravos e eventos em saúde pública de notificação compulsória em todo o território nacional e estabelece fluxo, critérios, responsabilidades e atribuições aos profissionais e serviços de saúde. Diário Oficial da União. 2011 Jan 25. [acesso em 2019 jul 12]. Disponível em: http://bvsms.saude. gov.br/bvs/saudelegis/gm/2011/prt0104_25_01_2011. html.

7. Brasil. Ministério do Desenvolvimento Social e Combate à Fome, Secretaria Nacional de Assistência Social. Política Nacional de Assistência Social - PNAS/2004. Brasília, DF: Ministério do Desenvolvimento Social e Combate à Fome; 2004.

8. Brasil. Presidência da República. Lei no ${ }^{\circ} 11.340$, de 7 de agosto de 2006. Cria Mecanismos para coibir a violência doméstica e familiar contra a mulher, nos termos do $98^{\circ}$ do art.226 da Constituição Federal, da Convenção sobre a Eliminação de Todas as Formas de Discriminação contra as mulheres e da Convenção Interamericana para Prevenir, Punir e Erradicar a Violência contra a Mulher; dispõe sobre a criação dos Juizados de Violência Doméstica e Familiar contra a Mulher; altera o Código de Processo Penal, o Código Penal e a Lei de execução Penal; e dá outras providências. [acesso em 2019 jul 12]. Disponível em: http://www.planalto.gov.br/ccivil_03/_Ato20042006/2006/Lei/L11340.htm.

9. Instituto Brasileiro de Geografia e Estatística. Censo 2010. [acesso em 2019 jun 30]. Disponível em: http:// censo2010.ibge.gov.br.

10. Brasil. Presidência da República, Secretaria de Políticas para as Mulheres. Plano Nacional de Políticas para as Mulheres. Brasília, DF: Secretaria de Políticas para Mulheres; 2013.

11. Viçosa. Conselho Municipal dos Direitos da Mulher (CMDM). Pacto Municipal de Enfrentamento à violência contra a Mulher. Viçosa; 2010.

12. Rezende DL, Andrade LVR. Rede não especializada de atendimento à mulher em situação de violência em Viçosa, Minas Gerais. Revista de Ciências Humanas. 2014; 14(1):207-225

13. Minayo MCS. O desafio do conhecimento: pesquisa qualitativa em saúde. São Paulo: Hucitec; 2008. 
14. Afonso ML, organizador. Oficinas em Dinâmica de Grupo: um método de intervenção psicossocial. São Paulo: Casa do Psicólogo; 2006.

15. Faria AAC, Ferreira Neto PS. Ferramentas do diálogo - qualificando o uso das técnicas do DRP: diagnóstico rural. Brasília, DF: MMA-IEB; 2006.

16. Kramer S. Entrevistas coletivas: uma alternativa para lidar com a diversidade, hierarquia e poder na pesquisa em ciências humanas. In: Freitas MT, Souza SJ, Kramer S, organizadores. Ciências humanas e pesquisa: leituras de Mikhail Bakhtin. São Paulo: Cortez; 2007.

17. Lourau R. A análise institucional. Petrópolis: Vozes; 2014.

18. Freire P. Pedagogia do oprimido. Rio de Janeiro: Paz e Terra; 2013.

19. Viçosa. Lei n ${ }^{\circ} 2.417$ de 21 de outubro de 2014. Dispõe sobre as diretrizes para atendimento integral e oportuno às mulheres em situação de violência, de acordo com as Leis Federais n ${ }^{0} 12.845 / 2013,10.778 / 2003$ e
11.340/2006 e o Decreto Presidencial no 7.958/2013 e dá outras providências. [acesso em 2019 ago 14]. Disponível em: https://leismunicipais.com.br/a/ $\mathrm{mg} / \mathrm{v} /$ vicosa/lei-ordinaria/2014/241/2417/lei-ordinaria-n-2417-2014-dispoe-sobre-as-diretrizes-para-atendimento-integral-e-oportuno-as-mulheres-em situacao-de-violencia-de-acordo-com-as-leis-federais-n-s-12845-2013-10-778-2003-e-11-340-2006-eo-decreto-presidencial-n-7-958-2013-e-da-outras-providencias.

20. Santos AP, Melo CM, Aguiar L. Protocolo municipal de atenção às mulheres em situação de violência. Viçosa: NIEG/UFV; 2015. [acesso em 2019 jul 30]. Disponível em: http://www.nieg.ufv.br/wp-content/uploads/ Protocolo-Municipal-de-Aten\%C3\%A7\%C3\%A3o-\%C3\%A0s-Mulheres-em-Situa\%C3\%A7\%C3\%A3o-de-Viol\%C3\%AAncia.pdf.

Recebido em 25/08/2019

Aprovado em 05/05/2020

Conflito de interesses: inexistente

Suporte financeiro: Conselho Nacional de Desenvolvimento

Científico e Tecnológico (CNPq) - Processo no 404877/2012-

5. Fundação de Amparo à Pesquisa do Estado de Minas Gerais

(Fapemig) - Processo no CDS - APQ-03625-12) 